\title{
Testing evolutionary tracks of Pre-Main Sequence stars: the case of HD113449
}

\author{
F. Cusano*, E. W. Guenther*, M. Esposito ${ }^{\dagger}$, M. Mundt**, E. Covino ${ }^{*}$ and \\ J. M. Alcalà \\ *Thüringer Landessternwarte Tautenburg, Sternwarte 5, D - 07778 Tautenburg \\ ${ }^{\dagger}$ Hamburger Sternwarte, Gojenbergsweg 112, 21029 Hamburg \\ ${ }^{* *}$ Max-Planck-Institut für Astronomie, Königstuhl 17, D - 69117 Heidelberg, Germany \\ $\ddagger$ Osservatorio Astronomico di Capodimonte, Salita Moiariello 16, 80131 - Napoli
}

\begin{abstract}
Evolutionary tracks are of key importance for the understanding of star formation. Unfortunately, tracks published by various groups differ so that it is fundamental to have observational tests. In order to do this, we intend to measure the masses of the two components of the Pre-Main Sequence (PMS) binary HD113449 by combining radial velocity (RV) measurements taken with HARPS, with infrared interferometric data using AMBER on the VLTI. The spectroscopic orbit that has already been determined, combined with the first AMBER measurement, allows us to obtain a very first estimation of the inclination of the binary system and from this the masses of the two stars. More AMBER measurements of HD 113449 are needed to improve the precision on the masses: in the ESO period P82 two new measurements are scheduled.
\end{abstract}

Keywords: Stars: binaries: general; fundamental parameters; Techniques: interferometric PACS: 97.80.Af, 97.80.Fk, 97.10.Nf

\section{INTRODUCTION}

The most fundamental parameter of a star is its mass which determines almost everything about its birth, life and death. For PMS stars this parameter is practically always derived by comparing the location of the star in the Hertzsprung-Russell Diagram (HRD) with theoretically calculated evolutionary tracks. Unfortunately, the evolutionary tracks published by various authors differ considerably due to the differences in the input physics [e.g. 3, 1, 5]. In order to test and calibrate the tracks it is necessary to determine the masses for a number of young stars. One possibility is to combine RV data and interferometric data in order to measure the masses of young stars in spectroscopic binary (SB) systems. In 1999, we thus initiated a spectroscopic survey for PMS binaries and detected 15 systems suitable for AMBER (see [7] for a description of the instrument) observations [4]. Unfortunately, all of these stars are too faint for AMBER at the present stage. In addition to these stars we found a young SB1 system in the course of an RV-survey with HARPS: HD113449. HD113449 is at a distance of only $22.1 \pm 0.6 \mathrm{pc}$ ([6]). The primary has a spectral type of G5V, and an equivalent width of the LiI6708 $\lambda$ line of $160 \mathrm{~m} \AA$. The secondary is presumably a late K or early M star. The binary thus consists of two post T Tauri stars, located in a zone of the HRD where the radiative core is supposed to form. This region of the HRD is of particular interest because the only stars where the masses have been determined are the two components of the binary system HD98800B ([2]), and these only match the evolutionary tracks if either a very low 

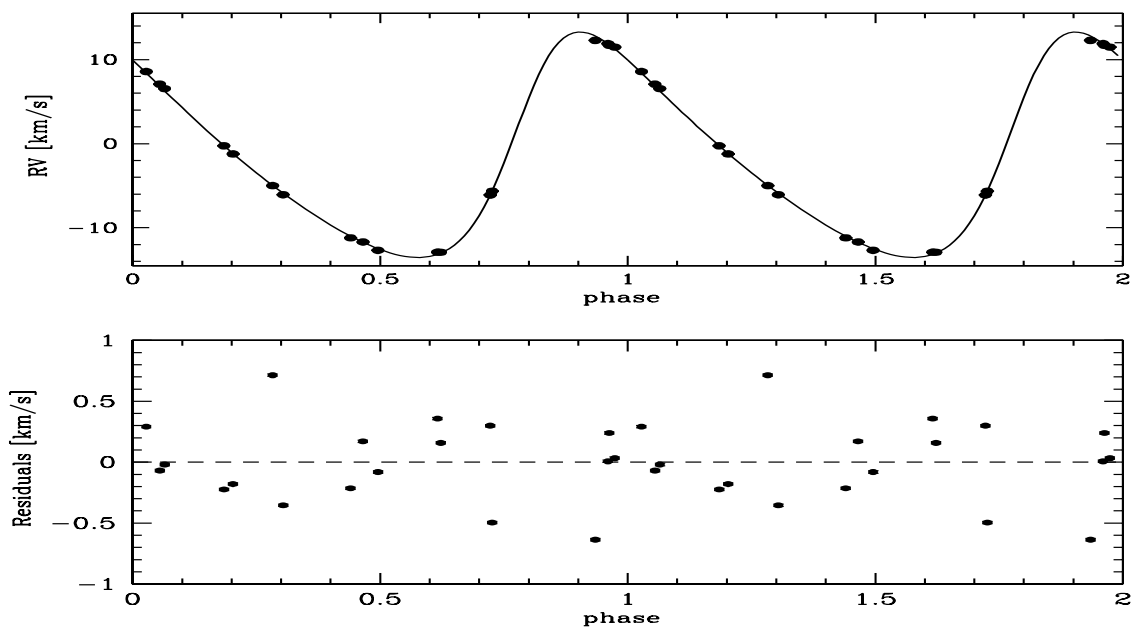

FIGURE 1. The phase-folded RV-measurements of HD113449. The orbital period is 216 days.

metallicity is assumed, or if the radiative core forms at an earlier stage than is presently assumed.

\section{OBSERVATIONS AND DATA REDUCTION}

Using HARPS, we have observed HD113449 for 2.5 years. The HARPS spectra cover the wavelength region from 378 to $691 \mathrm{~nm}$ at $\mathrm{R}=115000$. The HARPS pipeline was used in order to measure the RV of this star. We henceforth solved the SB1-orbit (Fig. 1), determining the orbital parameters given in Tab. 1. The inclination of the orbit of the binary with respect to the line of sight and the longitude of the ascending node $\Omega$ can not be derived from the RV-measurements. For this, the binary system has been observed also with AMBER, the near-infrared spectro-interferometric VLTI instrument. Observations have been performed the night between the 20-21st of March 2008. AMBER was used in Low-Resolution mode combining the light coming from the UT2, UT3 and UT4. In the same night, shortly before HD113449, a standard star, HD111998, of known diameter ( $\theta \sim 0.30$ mas) has been observed to calibrate the visibility. The data have been reduced at ESO using the AMBER-pipeline. The reduced data have been converted to ascii format running an automatic procedure in the ESOpipeline and a set of procedures specifically written by us have been used to calibrate the square visibility. In the first step we considered the calibrator to be unresolved on all the three baselines. This assumption leads to a small error on the calibrated visibility, especially for the largest baseline (UT1-UT3=77.9m). The larger scatter of the data on the right of Fig. 2 is presumably related to this assumption. As a next step we will correct for this. For HD113449 also an IR high-resolution spectra ( $R=100000,2236-2293 \mathrm{~nm})$ has been taken with CRIRES. Standard procedure have been used to reduce it. 
TABLE 1. Orbital elements of HD113449

\begin{tabular}{cc}
\hline element & value \\
& \\
\hline $\mathrm{P}$ & $215.9 \pm 0.1 \mathrm{~d}$ \\
$\mathrm{~T}_{0}[\mathrm{HJD}]$ & $2453411 \pm 1$ \\
$\gamma$ & $-1.79 \pm 0.02 \mathrm{~km} \mathrm{~s}^{-1}$ \\
$\mathrm{~K}_{1}$ & $13.40 \pm 0.02 \mathrm{~km} \mathrm{~s}^{-1}$ \\
$\mathrm{e}$ & $0.300 \pm 0.005$ \\
$\omega$ & $114.6 \pm 0.5^{\circ}$ \\
$\mathrm{a}_{1} \operatorname{sin~i}$ & $0.254 \pm 0.001 \mathrm{AU}$ \\
$\mathrm{f}(\mathrm{m})$ & $0.0467 \pm 0.0006 \mathrm{M}_{\odot}$ \\
\hline
\end{tabular}

\section{ANALYSIS AND RESULTS}

AMBER in the LR configuration produces spectral dispersed fringes along the $\mathrm{J}, \mathrm{H}$ and $\mathrm{K}$ bands. With a brightness of $\mathrm{J}=6.0, \mathrm{H}=5.7$, and $\mathrm{K}=5.5, \mathrm{HD} 113449$ is close to the limit of AMBER. The $\mathrm{S} / \mathrm{N}$ in $\mathrm{J}$, and $\mathrm{H}$ data is in fact so low that the data can not be used and our analysis thus is based on the K-band. To derive a first estimation of the inclination of the orbit and of the ascending node, $\Omega$, we compared the observations with a set of theoretical models. For this aim we wrote a software that simulates the visibility of a binary system given all the orbital parameters, the baselines and the epoch of observation. Given the distance to the system and the predicted radius both the stars are point sources. With this assumption the formula used for the visibility is simply: $V^{2}=\left(1+r^{2}+2 r \cos (\rho \mathbf{B} / \lambda)\right) /(1+r)^{2}$, where $r$ is the flux ratio between the two components, $\rho$ the angular separation, $\mathbf{B}$ the projected baseline and $\lambda$ the wavelength of observation. Fixing the parameters that were obtained from the RV-data, we vary just $i$ and $\Omega$, fixing also the flux in the K-band of the two stars assuming their spectral type. Considering that the primary is a G5V and the secondary an early M-star the flux ratio in the K-band is: $r=0.21 \pm 0.02$. Using a $\chi^{2}$ analysis we derive a value for the inclination of $\mathrm{i}=63 \pm 3^{\circ}$ and for the longitude of the ascending node $\Omega=102 \pm 8^{\circ}$. Fig. 2 shows the calibrated visibility squared vs. radius (in unit of Baseline/wavelength) of HD113449 during 45 minutes of observations. Also shown are the modeled visibility that best match the observations, from which we derive the inclination and the $\Omega$. From a first analysis of the CRIRES spectra (Fig. 3) we derive also a mass-ratio of primary to the secondary of $0.57 \pm 0.05$. Combining the value found for the inclination, the mass function and the mass ratio we estimate the masses to be $\mathrm{M}_{1}=0.88 \pm 0.13 \mathrm{M}_{\odot}$ and $\mathrm{M}_{2}=0.50 \pm 0.07 \mathrm{M}_{\odot}$ for the primary and the secondary, respectively. More observations with AMBER are needed to improve the derived orbital parameters. The AMBER and CRIRES data are also still being analysed.

\section{REFERENCES}

1. Baraffe, I., Chabrier, G., Allard, F., Hauschildt, P. H. 1998, A\&A, 337, 403

2. Boden, Andrew F., Sargent, Anneila I., Akeson, Rachel L. et al. 2005, ApJ, 635, 442

3. D’Antona, Francesca, \& Mazzitelli, Italo 1994, ApJS, 90, 467 


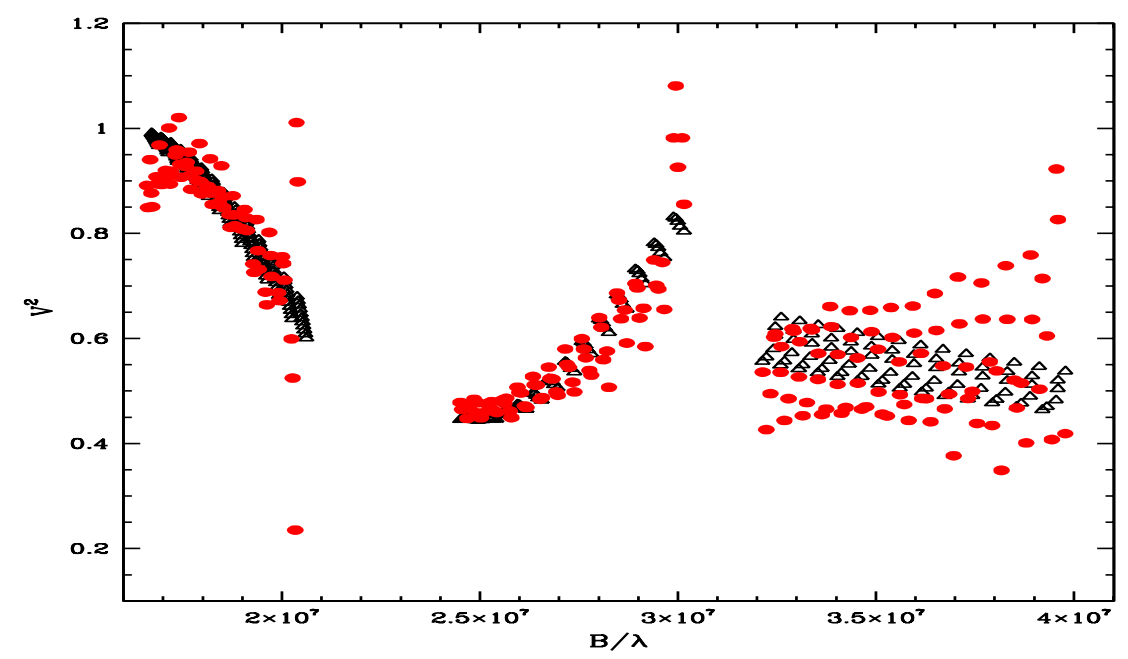

FIGURE 2. The red dots are the calibrated squared visibility of HD113449. Shown as black open triangles are models with $\mathrm{i} \sim 63$ and $\Omega \sim 102$ which match the observations best.

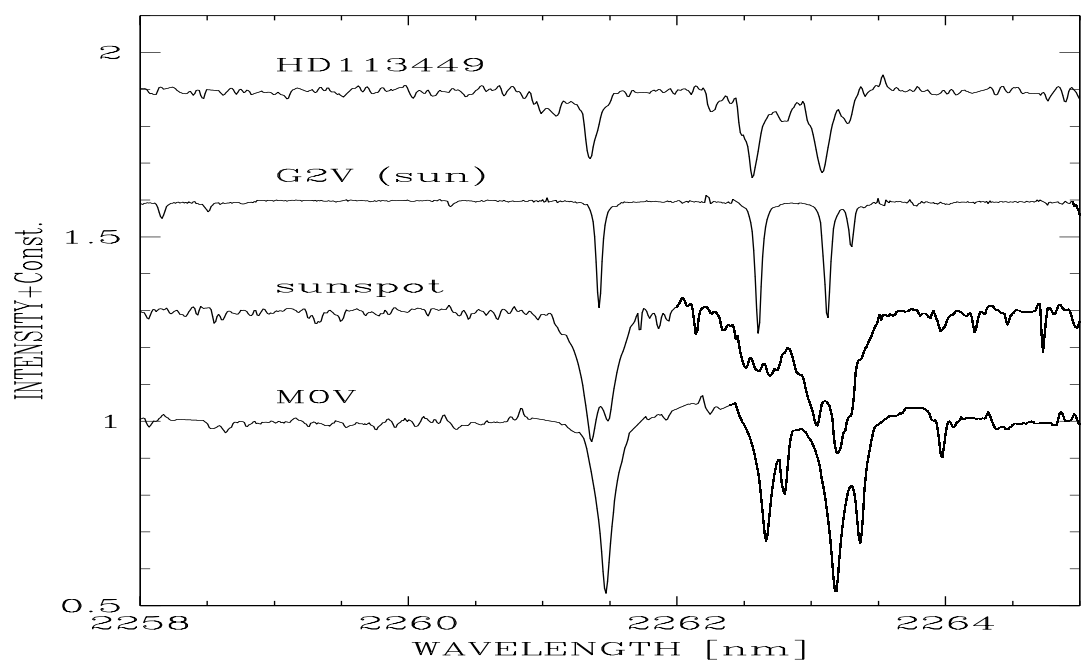

FIGURE 3. From top to bottom: a small part of the CRIRES spectrum of HD113449, FTS spectrum of the solar photosphere and a sunspot (M3V), and finally CRIRES spectrum of the M0V star GJ9847. Clearly visible in the spectrum of HD113449 are the lines of the G5V primary, and slightly blue-shifted to these the lines of the secondary. The lines in the spectrum of the sunspot (as well as GJ9847) are splitted into the Zeeman components.

4. Guenther, E. W., Esposito, M., Mundt, R., Covino, E., Alcalà, J. M., Cusano, F., Stecklum, B. 2007, A\&A, 467, 1147

5. Palla, Francesco, \& Stahler, Steven W. 1999, ApJ, 525, 772

6. Perryman, M.A.C., Lindegren, L., Kovalevsky, J. et al. 1997, A\&A, 323L, 49

7. Petrov, R. G., Malbet, F., Weigelt, G. et al. 2007, A\&A, 464, 1 